\title{
Expanding traumatic intracerebral contusion/hematoma
}

\author{
Yad R. Yadav, Abhijeet Basoor, Gaurav Jain, Adam Nelson \\ Neurosurgery Unit, NSCB Medical College, Jabalpur, MP, India
}

\begin{abstract}
Background and Aims: Delayed traumatic hematomas and expansion of already detected hematomas are not uncommon. Only few studies are available on risk factors of expanding hematomas. A prospective study was aimed to find out risk factors associated with such traumatic lesions. Materials and Methods: Present study is based on 262 cases of intracerebral hematomas / contusions out of which $43(16.4 \%)$ hematomas expanded in size. computerized tomography (CT) scan was done in all the patients at the time of admission and within 24 hours of injury. Repeat CT scan was done within 24 hours, 4 days and 7 days. Midline shift if any, prothrombin time, activated partial thromboplastin time, bleeding time, clotting time and platelet counts, Glasgow coma scale at admission and discharge and Glasgow outcome score at 6 months follow up were recorded. Results: Twenty six percent, 11.3 and $0 \%$ patients developed expanding hematoma in Glasgow Coma scale (GCS) of 8 and below, 9-12 and 13-15 respectively. The chances of expanding hematomas were higher in patients with other associated hematomas (17.4\%) as compared to isolated hematoma (4.8\%) (Fisher's exact results $P=0.216$ ). All the cases of expanding hematoma had some degree of midline shift and considerably higher proportion had presence of coagulopathy. The results of logistic regression analysis showed GCS, midline shift and coagulopathy as significant predictors for the expanding hematoma. Thirty nine patients $(90.7 \%)$ of the total expanding hematomas developed within 24 hours of injury. Conclusions: Enlargement of intracerebral hematomas is quite common and majority of them expand early after the injury. These lesions were common in patients with poor GCS, associated hematomas, associated coagulopathy and midline shift.
\end{abstract}

Key words: Delayed Intracerebral hematoma, head injury, secondary insult

\section{Introduction}

Post traumatic intracranial hematoma (ICH) can expand or develop late after head injury. ${ }^{[1-5]}$ Identification of risk factors, early diagnosis and proper treatment of such lesion are important for improving the prognosis of these patients. There are reports on delayed ICH, however very little literature is available on expansion of already existing intracerebral hematoma. ${ }^{[6,7]}$ We are reporting a prospective study of expansion of intracerebral hematoma.

\section{Materials and Methods}

A prospective study was carried out in neurosurgery unit of our institute from Jan.2002 to Dec 2003. Total of 326 post traumatic intracerebral contusions $(n=294) /$ hematomas $(n=32)$ were managed, out of these 47 patients were operated after first computerized tomography (CT) scan and were excluded from the study. 17 patients who died within 24 hours of admission (repeat scan could not be done in these cases) were also excluded from the study. Patients reported after 24 hours of injury were also excluded. The distinction between a contusion and an intracerebral hematoma was made by the proportion of blood in the lesion, if two thirds or more of the lesion was hematoma then it was considered as intracerebral hematoma. Increase in volume of 12.5 ec was considered as expansion of hematoma

A detailed history of injury, date and time of accident, age, sex, unconsciousness, ear nose throat bleeding, seizures, vomiting, headache, etc. was asked. A thorough neurological, systemic and general physical examination was done. Glasgow Coma scale (GCS) was obtained after resuscitation. CT scan was done in all the patients at the time of admission [Figures 1,3 and 5]. Repeat CT scan was done in all the patients of intracerebral contusions / hematomas within 24 hours of admission [Figures 2, 4, and 6]. Another CT scan was done on $4^{\text {th }}$ and $7^{\text {th }}$ day of the admission. 38 times CT scan was done outside the mentioned period due to clinical worsening out of these 29 were in none expanding and 9 in expanding group. Size of hematoma and midline shift was recorded. Hematoma volume was calculated using formula ABC/ 2 , where A was the greatest diameter of hemorrhage in single CT slice, $\mathrm{B}$ was the diameter $90^{\circ}$ to $\mathrm{A}$ and $\mathrm{C}$ is number of $\mathrm{CT}$ slice with hemorrhage multiplied by the slice thickness. Coagulation tests 


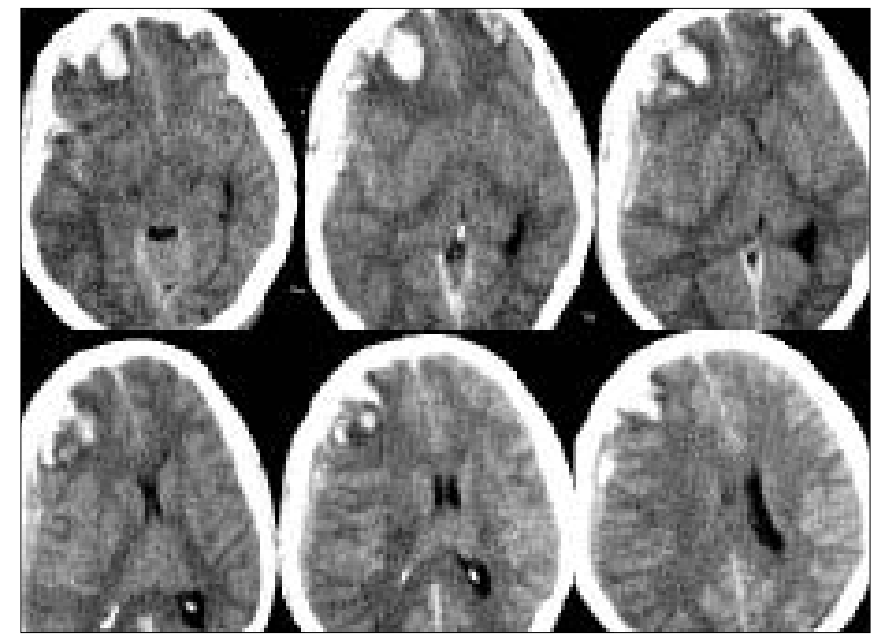

Figure 1: First CT scan of case 1 showing bifrontal ICH more on right side and an acute SDH in right fronto-temporo-parietal region

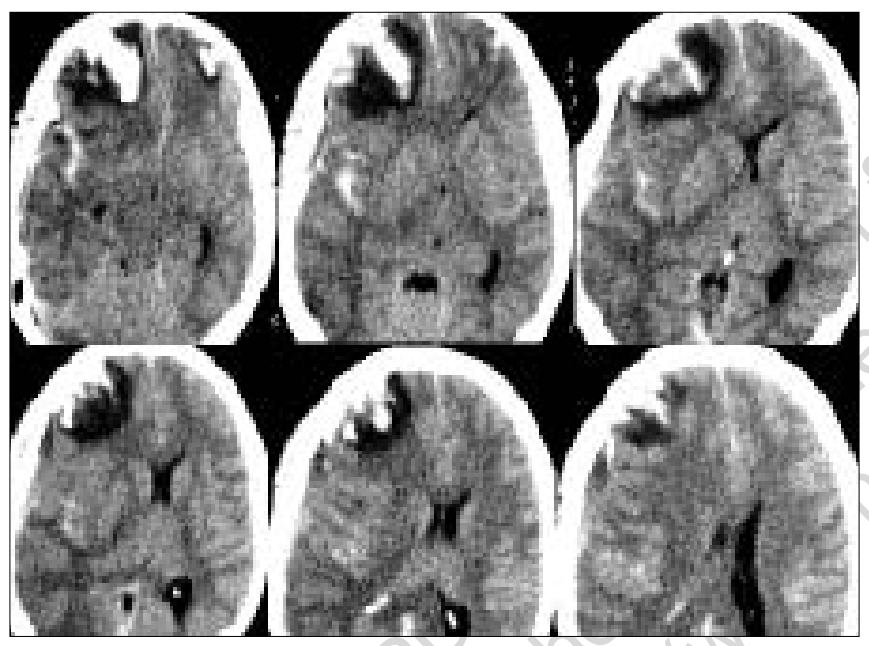

Figure 2: Second CT scan of case 1 showing no significant increase in $\mathrm{ICH}$

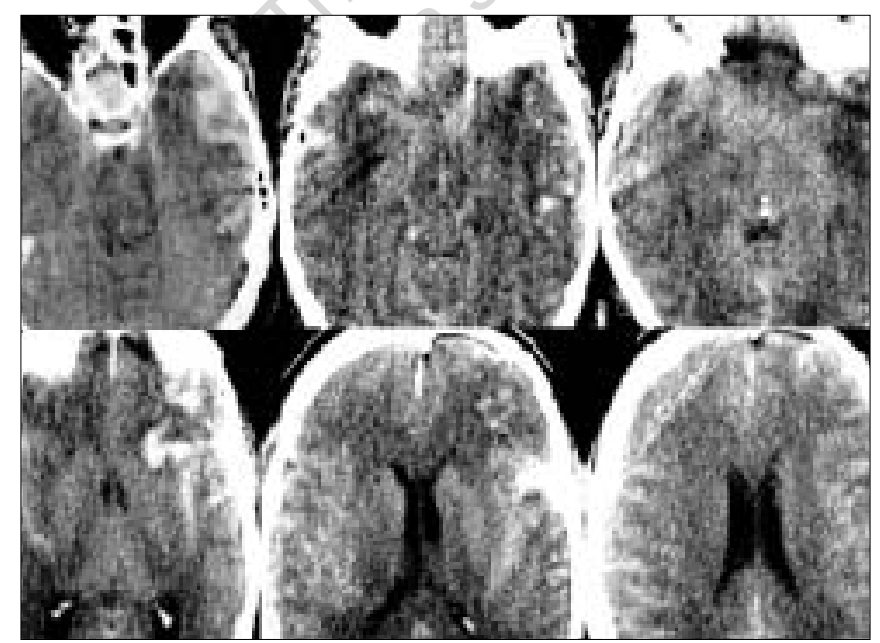

Figure 3: First CT scan of case 2 showing bilateral SAH involving fronto-temporo-parietal region and bilateral temporal contusions, more on left side

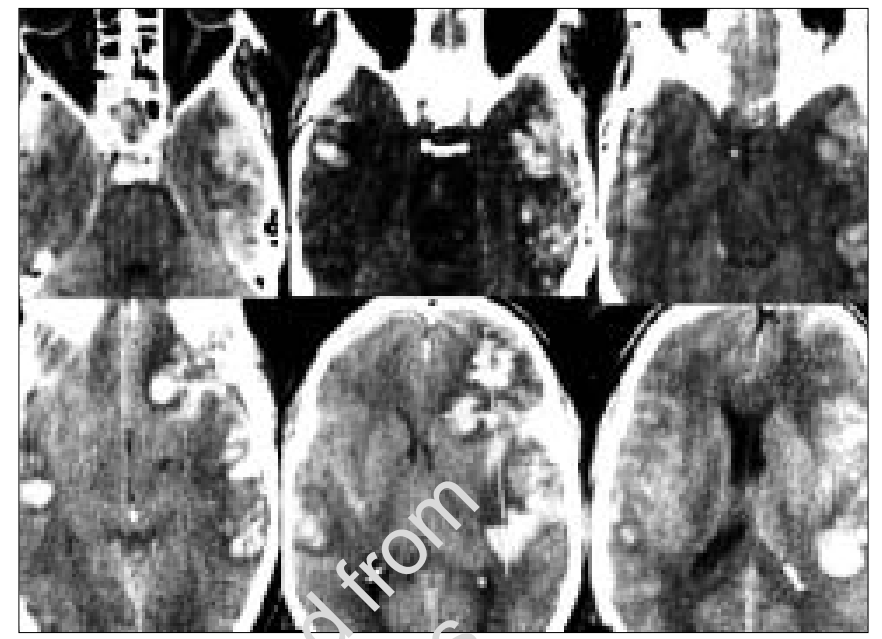

Figure 4: Second CT scan of case 2 showing significant increase in temporal contusions. This patient was operated after $2^{\text {nd }} \mathrm{CT}$ scan

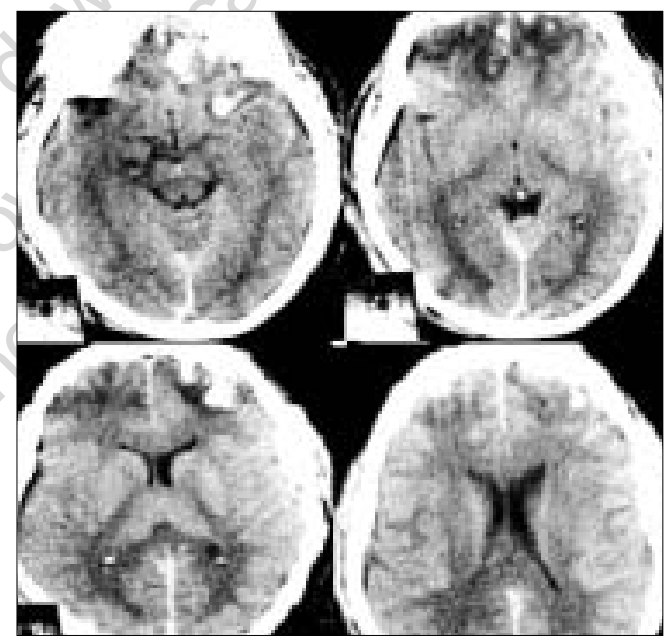

Figure 5: First CT scan of case 3 showing an acute SDH in left frontotemporo-parietal region and bifrontal contusions more on left side

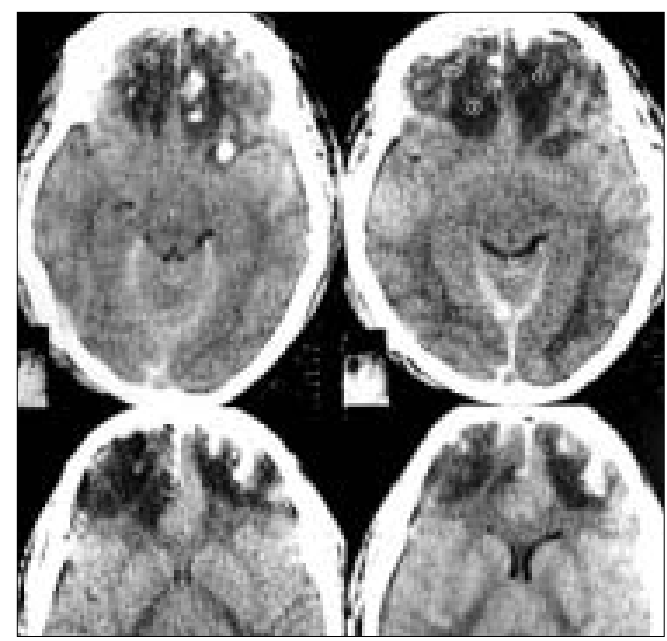

Figure 6: Second CT scan of case 3 showing significant increase in frontal contusions. This patient was treated conservatively, patient was conscious after second CT 
like prothrombin time (PT), activated partial thromboplastin time (APTT), bleeding time, clotting time and platelets counts were done in all the patients. Patients were diagnosed to have coagulopathy if PT and APTT were more than one and half times of the control and also when bleeding time or clotting time was more than upper limit and platelet count was less than lower limit of normal. All the patients with GCS of 8 and below were managed in ICU. All other relevant biochemical investigations were performed. Routine management of head injury patients was done. Results of treatment were recorded. Glasgow outcome scale was recorded at 6 months follow up.

The data for this study was analyzed using univariate, bivariate statistical analysis using fisher's exact test, Chi square test and a logistic regression analysis was also applied considering all these factors for the expanding hematoma.

\section{Results}

Out of 262 patients, $43 \mathrm{ICH}$ expanded in size. The analysis of 262 prospective cases of post traumatic intracerebral hematomas / contusions which was carried out in Neurosurgery unit of our institution is presented in this section. Majority $(66.5 \%)$ of the studied cases were in age below 40 years with a median age of 33 years. Age ranged from 2 to 80 years. Overall the mean age was 35.16 ( \pm 17.15 ) years. Male female ratio was 2.05 but the incidence of expanding hematoma in both the sex was equal having insignificant difference $(P>0.05)$. Initial ICH volume ranged from 4-18 cubic cm [ce] with a mean value of $12.06( \pm 3.70) \mathrm{ce}$ and this was increased from 12.5 to $58 \mathrm{ec}$ with a mean value of 33.13 $( \pm 10.59)$ ce and this increase in ICH volume was statistically significant. $(P<0.001) 34$ patients deteriorated in GCS from 13 . The deterioration in GCS was from 1-3 in operated group as compared to 1-2 in conservatively managed groups. Increase in midline shift in the operated groups was 2-5 $\mathrm{mm}$ while it was 1-3 $\mathrm{mm}$ in conservatively managed groups.

As depicted in Table 1, out of 262 cases 104 (39.7\%) cases were observed with GCS $\leq 8,141(53.8 \%)$ with GCS 9-12 and $17(6.5 \%)$ with GCS $13-15$ and their corresponding findings of expanding hematoma were $26.0,11.3$ and $0.0 \%$ respectively. The incidence of expanding hematoma in cases with lower GCS was significantly higher. A linear trend was observed in GCS and expanding hematoma i.e., the cases with lower GCS have higher chances of expanding hematoma. $(P<0.001)$ The impact of GCS shows its direct correlation with the development of hematomas.

There were 21(8.0\%) patients with single ICH/ contusion as compared to $241(92.0 \%)$ with multiple lesions like acute subdural hematoma (SDH) in $134(55.6 \%)$, other ICH in 51 $(21.2 \%)$, extra-dural hematoma in $9(3.7 \%)$, SDH and other ICH in $47(19.5 \%)$ patients. $1(4.8 \%)$ out of 21 isolated ICH [without any other hematomas] developed expanding hematomas as compared to 42 patients $(17.4 \%)$ with other associated hematomas $[n=241]$ but this difference was statistically not significant (fisher exact test results $P=0.216$ ). Twenty four patients (17.9\%) out of $134 \mathrm{ICH}$ associated with acute SDH, 10 $(19.6 \%)$ patients out of 51 multiple ICH and $8(17.0 \%)$ out of 47 both SDH and ICH developed expanding hematomas. This difference in incidence of expanding hematoma among various associated hematomas is not statistically significant $\left(\chi^{2}=0.12\right.$; $P>0.05)$.

The study of midline shift showed that there were $249 \mathrm{ICH} /$ contusions with midline shift while in 13 cases there was no midline shift observed, out of the cases with midline shift $17.3 \%(n=43)$ developed expanding hematoma while none of the patients without midline shift developed expanding hematomas.

This difference was also statistically insignificant (fisher exact test results $P=0.135$ ) while this was one of the significant factor in the logistic regression analysis. There were 41, 87, 108 and 13 patients with midline shift of less than $3 \mathrm{~mm}, 3$ to less than $5 \mathrm{~mm}$, 5 to less than $10 \mathrm{~mm}$ and more than $10 \mathrm{~mm}$ respectively, out of these $4(9.7 \%), 14(16.1 \%), 21(19.4 \%)$ and $4(30.8 \%)$ developed expanding hematomas respectively. $\left(\chi^{2}=3.72\right.$; $P>0.05$ at $3 \mathrm{df}$ )

Coagulopathy was directly related to expanding hematoma. Coagulopathy was seen in 39 patients of ICH. Out of these 41.0 percent developed expanding hematoma while the incidence of expanding hematoma was only $12.1 \%$ where the coagulopathy was absent and statistically this was highly significant $(P<0.001)$.

Surgeries were required in 37 cases out of 43 expanding hematomas. $19(51.3 \%)$ patients in operative group died, while there was no mortality in conservatively treated group. However they had better grade with small ICH. 4 patients (one operative and 3 conservative group) could not be followed up out of 24 survivors. All three patients of conservative group who could be followed up had good recovery while 5, 5, 4 and 3 patients of operated group had good recovery, mild, moderate and severe disability (35\% moderate to severe disability) at 6 months follow up.

The location of the ICH/ contusions were 106 in frontal region, 134 in temporal, 12 in parietal region and 10 in more than one region, out of these 18 (17.0\%), $23(17.2 \%), 1$ (8.3\%),1 (10.0\%) in frontal, temporal, parietal and more than one region developed expanding hematomas respectively. $39(90.7 \%)$ expanding hematomas developed within 24 hours of injury while the rest $2(4.6 \%)$ patients each developed ICH within 4 days and 7 days.

The result of logistic regression analysis showed positive value for the Cox and Snell R square $\left(\mathrm{R}^{2}=0.216\right)$ and found that GCS $(P<0.001)$, midline shift $(P<0.001)$ and coagulopathy $(P<0.003)$ were highly significant predictors for the expanding hematoma.

\section{Discussion}

We used the term expanding traumatic intracerebral contusion / hematoma which expand after the initial detection on first CT scan while in cases of delayed hematomas, initial CT scan was normal. The incidence of expanding hematoma was $16.4 \%$ in our 
series while it was $42.3 \%$ in Oertel series ${ }^{[6]}$ and $(68.2 \%)$ in Lobato $^{[7]}$ series. The risk factors of delayed hematomas like coagulopathy, presence of preexisting intracerebral contusion / hematoma, traumatic vessel injury, subdural hematoma and development of hematoma after treatment of raised intracranial pressure are described. ${ }^{[1-12]}$ Similar mechanism of development of expansion of hematoma may exist.

Delayed hematomas are known to be associated with coagulation disorder. $47.6 \%$ patients of traumatic sub-arachnoid hemorrhage with coagulopathy at admission developed delayed hematoma. ${ }^{[1]}$ In our series also $41 \%$ of ICH with coagulopathy developed expansion of hematoma. Delayed and recurrent intracranial hematomas were found to be related to disseminated intravascular clotting and fibrinolysis. ${ }^{[3]}$ These were also reported in patients taking oral anticoagulants. ${ }^{[2]}$ Delayed hematomas were commonly associated with SDH and underlying contusion / hematoma. ${ }^{[13]}$ Expanding hematomas were also more common in ICH associated with other hematomas as compared to isolated ICH in our series although the difference was insignificant. It could be due to venous infarct developing in the contused brain region. Injured draining veins responsible for the acute $\mathrm{SDH}$, could also be responsible for delayed/ expansion of hematoma.

Delayed intracerebral hematoma can also develop in patients with raised intracranial pressure. Treatment of such patients by either surgery or medicine relieves temponade effect which can result in bleeding from injured vessel in area of contused brain. ${ }^{[10,12,14]}$ Expanding hematomas were more common in frontal and temporal region. Vascular injury like angionecrosis, intravascular thrombosis could be responsible for expanding hematoma. ${ }^{[5,15]}$ The chances of expanding hematomas were higher in patients with other associated hematomas (17.4\%) as compared to isolated hematoma $(4.8 \%)$ (Fisher's exact results; $P=0.216$ ) in our series similar observations were made in other series. ${ }^{[16}$

Intracerebral haematomas with midline shift were also associated with higher incidence of expansion of hematoma as compared to haematomas without midline shift in our series but the differences were insignificant in fisher's exact results due to small number however it was one of the predictor observed significant in the logistic regression analysis.

Expanding hematomas developed more commonly in patients in poor GCS and with other associated hematomas in our series; this was also observed in other series. ${ }^{[16,17]}$ These were also more commonly associated with midline shift as compared to patients without midline shift. Age was not found to be risk factor in this study and in Stein et al series, ${ }^{[16]}$ while it was found as a risk factor in other series. ${ }^{[6]}$ They also found sex as a risk factor which was also not seen in our series or in any other series.

Coagulopathy was also found to be significant predictors for the expanding hematoma in our series; this was also observed in other series. ${ }^{[6,16,17]}$ Thirty nine patients $(90.7 \%)$ of the total expanding hematomas developed within 24 hours of injury in our study, similarly majority $(80.7 \%)$ of delayed hematomas were noted within 48 hours after injury ${ }^{[18]}$ and within 72 hours of injury. ${ }^{[8]}$ Okada $^{[19]}$ et al observed that the times, when the formations of traumatic intracerebral hematomas were judged as completed showed two peaks: within 6 hours after the trauma and 12 to 24 hours after the trauma. Oertel $e t a l^{[6]}$ also observed progressive hemorrhage in $48.6 \%$ of patients who underwent scanning within 2 hours of injury.

Expanding hematoma can be diagnosed by repeat CT scan. Lobato et $a l^{[7]}$ advised serial CT scanning at 2-4, 12, 24, 48 and 72 hours after injury. Near infrared spectroscopy can also detect such lesions much earlier. ${ }^{[20]}$ Prevention of these hematomas can be done by correction of risk factors like coagulopathy, treatment of raised intracranial pressure etc. Early diagnosis should be done by timely CT scan when patient is at risk like patient with cerebral contusion / hematoma, associated with other hematomas, raised ICP with midline shift, coagulopathy and patients in poor GCS.

Treatment of such lesions consists of surgical removal when patient is in altered sensorium, with large clot and with midline shift. Small lesions in conscious patient can be managed by conservative method. Prognosis is poor with 50- $60 \%$ mortality and increased morbidity in majority of patients.

\section{Acknowledgement}

Authors are thankful to Mr. Arvind Kavishwar, Bio Statistician, ICMR Jabalpur for his statistical assistance in this work.

\section{References}

1. Sawauchi S, Yuhki K, Abe T. The relationship between delayed traumatic intracerebral hematoma and coagulopathy in patients diagnosed with atraumatic subarachnoid hemorrhage. No Shinkei Geka 2001;29:131-7.

2. Halatsch ME, Markakis E. Phenoprocoumon, head trauma and delayed intracerebral hemorrhage. Funct Neurol 1999;14:155-7.

3. Kaufman HH, Moake JL, Olson JD, Miner ME, duCret RP, Pruessner JL, et al. Delayed and recurrent intracranial hematomas related to disseminated intravascular clotting and fibrinolysis in head injury. Neurosurgery 1980;7:445-9.

4. Mlay SM. Delayed intracranial hematomas. East Afr Med J 1990;67:717-9

5. Zhang G, Wang D, Li Q, Chen H, Shan Y. Encephalic morphologic changes due to delayed traumatic intracerebral hematoma. Fa Yi Xue Za Zhi 1998;14:1-3, 61.

6. Oertel M, Kelly DF, McArthur D, Boscardin WJ, Glenn TC, Lee JH, et al. Progressive hemorrhage after head trauma: Predictors and consequences of the evolving injury. J Neurosurg 2002;96:109-16.

7. Lobato RD, Alen JF, Perez-Nunez A, Alday R, Gomez PA, Pascual B, et al. Value of serial CT scanning and intracranial pressure monitoring for detecting new intracranial mass effect in severe head injury patients showing lesions type 1-11 in initial CT scan. Neurocirugia (Astur) 2005;16:217-34

8. Ninchoji T, Uemura K, Shimoyama I, Hinokuma K, Bun T, Nakajima S. Traumatic intracerebral hematomas of delayed onset. Acta Neurochir (Wien) 1984;71:69-90. Borthne A, Sortland O, Blikra G. Head injury with delayed intracranial hemorrhage. Tidsskr Nor Laegeforen 1992;112:3425-8.

10. Kojima N, Tamaki N, Matsumoto S, Fujiwara M. Delaved onset of intraventricular hemorrhage following removal of acute subdural hematoma- Case report. Neurol Med Chir (Tokyo) 1991;31:165-8

11. Xia GD. Diagnosis and treatment of delayed intracranial hematoma. Zhonghua Wai Ke Za Zhi 1989;27:674-5,702.

12. Hirsh LF. Delayed traumatic intracerebral hematomas after surgical decompression. Neurosurgery 1979;5:653-5.

13. Young-Su P, Ishikawa .J. Wide craniotomy-small dural incisions and intentionally delayed removal of intracerebral contusional hemorrhage for acute subdural hematoma. No Shinkei Geka 1997;25:1081-9.

14. Erol FS, Kaplan M, Topsakal C, Ozveren MF, Tiftikei MT. Coexistence of rapidly resolving acute subdural hematoma and delayed traumatic intracerebral hemorrhage. Pediatr Neurosurg 2004;40:238-40.

15. Stein SC, Graham DI, Chen XH, Smith DH. Association between intravascular micro thrombosis and cerebral ischemia in traumatic brain injury. Neurosurgery 
2004;54:687-91

16. Stein SC, Spettell C, Young G, Ross SE. Delayed and progressive brain injury in closed-head trauma: Radiological demonstration. Neurosurgery 1993;32:25-31.

17. Stein SC, Spettell CM. Delayed and progressive brain injury in children and adolescents with head trauma. Pediatr Neurosurg 1995;23:299-304

18. Zhang G, Wang D, Cheng D. The delayed traumatic intracerebral haematomas. Zhonghua Wai Ke Za Zhi 1995;33:430-2.

19. Okada T. Clinical aspects of traumatic intracerebral hematomas. Pathogenesis of delayed traumatic intracerebral hematomas. Nippon Ika Daigaku Zasshi
$1989 ; 56: 545-58$

20. Gopinath SP, Robertson CS, Contant CF, Naravan RK, Grossman RG, Chance B. Early detection of delayed traumatic intracranial hematomas using near infrared spectroscopy. J Neurosurg 1995;83:438-44.

Accepted on 20-09-2006

Source of Support: Nil, Conflict of Interest: None declared.

\section{Author Help: Reference checking facility}

The manuscript system (www.journalonweb.com) allows the authors to check and verify the accuracy and style of references. The tool checks the references with PubMed as per a predefined style. Authors are encouraged to use this facility before submitting articles to the journal.

- The style as well as bibliographic elements should be $100 \%$ accurate to get the references verified from the system. A single spelling error or addition of issue number / month of publication will lead to error to verifying the reference.

- $\quad$ Example of a correct style

Sheahan P, O'leary G, Lee G, Fitzgibbon J. Cystic cervical metastases: Incidence and diagnosis using fine needle aspiration biopsy. Otolaryngol Head Neck Surg 2002;127:294-8.

- Only the references from journals indexed in PubMed would be checked.

- $\quad$ Enter each reference in new line, without a serial number.

- $\quad$ Add up to a maximum 15 reference at time.

- If the reference is correct for its bibliographic elements and punctuations, it will be shown as CORRECT and a link to the correct article in PubMed will be given.

- If any of the bibliographic elements are missing, incorrect or extra (such as issue number), it will be shown as INCORRECT and link to possible articles in PubMed will be given. 